\title{
PCSK9 genetic variants and cognitive abilities: a large-scale Mendelian randomization study
}

Donald M. Lyall1', Joey Ward ${ }^{1}$, Maciej Banach², George Davey Smith ${ }^{3,4}$, Jason G. Gill ${ }^{5}$, Jill P. Pell1, Michael V. Holmes ${ }^{3,6-8}$, Naveed Sattar ${ }^{5}$

\author{
${ }^{1}$ Institute of Health and Wellbeing, University of Glasgow, Scotland, UK \\ ${ }^{2}$ Department of Hypertension, Medical University of Lodz, Lodz, Poland \\ ${ }^{3}$ Medical Research Council Integrative Epidemiology Unit, University of Bristol, Bristol, UK \\ ${ }^{4}$ School of Social and Community Medicine, University of Bristol, Bristol, UK \\ Institute of Cardiovascular and Medical Sciences, University of Glasgow, Scotland, UK \\ ${ }^{6}$ Medical Research Council Population Health Research Unit at the University \\ of Oxford, Oxford, UK \\ ${ }^{7}$ Clinical Trial Service Unit and Epidemiological Studies Unit (CTSU), Nuffield \\ Department of Population Health, University of Oxford, Oxford, UK \\ ${ }^{8}$ National Institute for Health Research, Oxford Biomedical Research Centre, \\ Oxford University Hospital, Oxford, UK
}

Submitted: 1 September 2020

Accepted: 17 September 2020

Arch Med Sci 2021; 17 (1): 241-244

DOI: https://doi.org/10.5114/aoms/127226

Copyright $\odot 2020$ Termedia \& Banach

\section{Abstract}

Introduction: PCSK9 inhibitors lower low-density lipoprotein (LDL) cholesterol and are efficacious at reducing vascular disease, however questions remain about potential effects on cognitive function.

Methods: We examined the association of genetic variants in PCSK9 with continuous measures of cognitive ability in UK Biobank. Six independent polymorphisms in PCSK9 were used in up to 337,348 individuals.

Results: The PCSK9 allele score was associated with a lower risk of CHD, and weakly with worse log reaction time.

Conclusions: We are unable to rule out meaningful associations of PCSK9 genetic variants with cognition, emphasising the potential need for continued pharmacovigilance for patients currently treated with PCSK9 inhibitors.

Key words: low-density lipoprotein, cardiovascular genomics, epidemiology.

Therapeutic modification of atherogenic lipoproteins by statins [1], ezetimibe [2] and Proprotein convertase subtilisin/kexin type 9 (PCSK9) inhibition [3] is an effective strategy to reduce the risk of cardiovascular disease (CVD), principally by lowering non-HDL-cholesterol [4]. PCSK9 regulates LDL-cholesterol (LDL-C) through hepatic expression of LDL receptors. PCSK9 inhibitors are licensed as LDL-C lowering agents with excellent efficacy and evidence of cardiovascular benefits: the FOURIER trial [3] reported that a monoclonal antibody to PCSK9 that lowered LDL-C by $59 \%(\sim 56 \mathrm{mg} / \mathrm{dl})$ led to a $15 \%$ reduction in the risk of a composite of cardiovascular death, myocardial infarction, stroke, hospitalization for unstable angina or coronary revascularization in patients with established atherosclerotic vascular disease. However, early phase 3 studies showed a potential excess of neurocognitive adverse effects [5], leading the US FDA to instruct pharmaceutical companies to assess potential neurocognitive side effects of PCSK9 inhib-

\author{
Corresponding authors: \\ Prof. Naveed Sattar \\ Institute of Cardiovascular \\ and Medical Sciences \\ BHF Glasgow \\ Cardiovascular \\ Research Centre \\ University of Glasgow \\ 126 University Place \\ Glasgow, G12 8TA \\ Phone: +44 1413303419 \\ E-mail: Naveed.Sattar@ \\ glasgow.ac.uk \\ Dr. Michael Holmes \\ Medical Research \\ Council Population \\ Health Research Unit \\ University of Oxford \\ Old Road Campus \\ Oxford, OX3 7LF \\ Phone: +44 1865743644 \\ E-mail: Michael.Holmes@ \\ ndph.ox.ac.uk
}


itors. While, reassuringly, no excess risk was noted in the FOURIER trial [3] or its substudy with detailed cognitive measures [6], these studies cannot fully exclude potential adverse effects from longer-term use of PCSK9 inhibitors. An orthogonal approach to obtain reliable information is to exploit genetic variants that mimic pharmacological inhibition of PCSK9. Naturally occurring variation in the gene encoding a drug target can be used to gauge insight on long-term effects of therapeutic modification [7]. So-called drug-target Mendelian randomization [8] exploits the characteristics of the genotype for the reliable estimation of both intended and unintended consequences of therapeutic modification of a drug target, as previously demonstrated [9-12].

Methods. We used four measures of cognitive ability from UK Biobank (UKB) data: two from baseline (2006-2010): fluid reasoning measured in 160,130 individuals (with genetic data, prior to exclusions listed below) and reaction time in 482,187 as they showed good intra-participant longitudinal reliability in $n=19,999$ participants [13], and two measured in 2014-2015 via the internet: trail making test (TMT) A (processing speed) in 100,587 and $B$ (speed plus executive function) in 100,610 , and digit symbol coding (executive function) in 115,933 . TMT and reaction time scores were log-transformed due to a positive skew. All measures were standardized to Z-scores.

Six independent single nucleotide polymorphisms (SNPS) $\left(R^{2}<0.15\right)$ in PCSK9 (referent allele frequencies rs2479394 $\mathrm{A}=0.72$; rs 11206510 $C=0.19 ; r s 2479409 A=0.65 ; r s 10888897 \mathrm{~T}=$ 0.39 ; rs7552841 C = 0.63; rs562556 G = 0.18); orientated so that the effect allele associated with a lower LDL-C were used as genetic variants to proxy therapeutic inhibition of PCSK9. SNPS were selected from the paper by Ference et al. in NEJM [13]. In the paper by Ference et al. [14], seven PCSK9 SNPs were used, however two were found to be moderately correlated (rs2479409 and rs2149041; $R^{2}=0.37$ ). We therefore removed the SNP with the weaker association with LDL-C (rs2149041) as defined by the $p$-value [14] leaving six SNPs (with pair-wise LD $R^{2}<0.15$ ) described above. The LDL-C association of each of the six PCSK9 SNPs from the Global Lipids Genetics Consortium [15] was used to construct a weighted PCSK9 allele score.

Ethical approval. This secondary-data analysis study was conducted under the generic approval from the NHS National Research Ethics Service (approval letter dated $17^{\text {th }}$ June 2011, ref. 11/ NW/0382). Written informed consent was obtained from all participants in the study (consent for research, by UK Biobank).

Statistical analysis. Linear regression analyses used the four cognition traits as dependent variables, the weighted PCSK9 score as the independent variable, adjusted for age, sex, GWAS array, and 10 principal components (as provided by UKB). To mimic pharmacological modification of PCSK9, we report results of the PCSK9 allele score scaled to the $50 \mathrm{mg} / \mathrm{dl}$ lower LDL-C achieved in FOURIER. We compared estimates of the PCSK 9 allele score with cognition traits to those from $A P O E$ e4 dosage (excluding rare $A P O E$ e2/e4), $A P O E$ e4/e4 vs. e3/e3 homozygosity; current vs. never smoking, and 5 -years of increased cross-sectional age with cognitive traits. As a further positive control, we tested the association of the PCSK9 allele score with the risk of CHD in UK Biobank (defined as self-reported physician-diagnosed myocardial infarction and angina).

We excluded participants with non-white British ancestry, self-report vs. genetic sex mismatch, putative sex chromosomal aneuploidy, excess heterozygosity, and missingness rate $>0.1$. We removed one random participant in cases where two individuals were first cousins or closer. Stata v14 and PLINK v1.90 were used for analyses.

Data availability statement. UK Biobank is an open access resource available to verified researchers upon application (http://www.ukbiobank. ac.uk/). Analysis syntax is available upon request.

Results. The PCSK9 allele score scaled to a $50 \mathrm{mg} / \mathrm{dl}$ lower LDL-C was associated with a lower risk of CHD in UKB (comprising 15,284 cases of myocardial infarction and angina in 338,852 individuals; $\mathrm{OR}=0.73 ; 95 \% \mathrm{Cl}: 0.60-0.90, p=0.003$ ).

We next investigated the associations of six variants in PCSK9 scaled to $50 \mathrm{mg} / \mathrm{dl}$ lower LDL-C in 109,870 individuals with measures of fluid reasoning, 337,348 with reaction time, 73,044 with processing speed (TMT A), 73,063 with processing speed plus executive function (TMT B), and 82,012 with digit symbol coding (executive function) (Figure 1).

Orientated to a $50 \mathrm{mg} / \mathrm{dl}$ lower LDL-C (i.e. mimicking pharmacological inhibition of PCSK9), the PCSK9 allele score was nominally associated with log reaction time $(0.04$ SDs higher log reaction time; $95 \% \mathrm{Cl}: 0.002-0.079 ; p=0.038)$. For fluid reasoning, the scaled $P C S K 9$ allele score had wide $95 \% \mathrm{Cl}(-0.08,0.07)$ that included the estimates for the association of 5 years additional age $(-0.05$ SDs, $95 \% \mathrm{Cl}$ : $-0.06,-0.05)$. Similar patterns were identified for all other cognition traits, meaning that despite the large sample size, the imprecision around the estimates obtained from the PCSK 9 allele score meant that we could not exclude a similar magnitude of effect of genetic inhibition of PCSK9 to that seen with the positive controls, including APOE e4 or smoking status for any of the individual cognition traits (Figure 1). Notably, point estimates for the association of the PCSK9 allele 




Figure 1. Association of a PCSK9 allele score (in gray) scaled to $50 \mathrm{mg} / \mathrm{dl}$ lower LDL-cholesterol and other selected exposures (in black) with cognitive traits in UK Biobank

score and all cognitive ability end-points were on the harmful side of unity. In sensitivity analyses, removal of participants that self-reported a neurological condition ( $~ 5 \%$ of the dataset) [13] did not alter the findings, nor did substituting rs2479409 for rs2149041 in the PCSK9 allele score.

Discussion. In this large-scale analysis of individuals from the general population, we used naturally occurring genetic variants in PCSK9 to gauge insight into the effect of lifelong lowering of LDL-C through inhibition of PCSK9 and its association with cognition abilities. Using available data in UKB, we were not able to provide definitive evidence on the relationship of PCSK 9 genetic variants with cognition traits. While this may have arisen due to lack of power, we note firstly that we were able to show associations of the PCSK9 allele score with the risk of prevalent self-reported CHD, and secondly robust associations of conventional risk factors (e.g. age and smoking) and genetic variants (e.g. APOE e4) with the cognitive traits. These observations suggest validity of the PCSK9 genetic score as an instrument, and suf- ficient statistical power for detecting association with relevant traits.

Our findings add to previous studies of genetically-estimated PCSK9 and cognition: Schmidt et al. [16] reported no significant association in nine cohorts, three of which indexed cognition with a screening tool (Mini-mental state exam) rather than normative-range tests as per here; while Rao et al. reported no effect on a single cognitive test (Trail-making; processing speed and executive function) [17]. The cognitive tests used here were novel, brief and bespoke to UK Biobank baseline assessment [13], and therefore examinations of cognitive assessments which are based on more traditional and validated tasks, may be informative. Such analyses may also test for potential age- and sex-specific associations.

While the imprecision makes it challenging to draw firm conclusions about an effect (or lack thereof) of life-long LDL-cholesterol lowering by PCSK9 inhibition on cognition, our data highlight the need for additional large-scale genetic analyses. In parallel, continued pharmacovigilance is 
potentially needed for patients currently treated with PCSK9 inhibitors.

\section{Acknowledgments}

$\mathrm{MVH}$ works in a unit that receives funds from the University of Oxford and the UK Medical Research Council and is supported by the British Heart Foundation Intermediate Clinical Research Fellowship (FS/18/23/33512) and the National Institute for Health Research Oxford Biomedical Research Centre. DML is supported by the American Psychological Foundation. George Davey Smith works in the Medical Research Council Integrative Epidemiology Unit at the University of Bristol MC_UU_00011/1. The funders had no role in study design, decision to publish, or preparation of the manuscript. UK Biobank was established by the Wellcome Trust medical charity, Medical Research Council (MRC), Department of Health, Scottish Government and the Northwest Regional Development Agency. We are grateful to UK Biobank participants.

This research was conducted using UK Biobank application 17689; we are grateful to UK Biobank staff and participants.

Naveed Sattar MD PhD FMedSci ( ${ }^{\star}$ Joint senior authors)

\section{Conflict of interest}

The Clinical Trial Service Unit and Epidemiological Studies Unit at the University of Oxford (MVH) has received research grants from Abbott/ Solvay/Mylan, AstraZeneca, Bayer, GlaxoSmithKline, Merck, Novartis, Pfizer, Roche, and Schering. $\mathrm{MVH}$ has collaborated with Boehringer Ingelheim in research, and in accordance with the policy of the Clinical Trial Service Unit and Epidemiological Studies Unit (University of Oxford), did not accept any personal payment. NS has consulted for AstraZeneca, Bristol-Myers Squibb, Amgen, Sanofi, and Boehringer Ingelheim. All other authors have nothing to report.

\section{References}

1. Collins R, Reith C, Emberson J, et al. Interpretation of the evidence for the efficacy and safety of statin therapy. Lancet 2016; 388: 2532-61.

2. Cannon CP, Blazing MA, Giugliano RP, et al. Ezetimibe added to statin therapy after acute coronary syndromes. N Engl J Med 2015; 372: 2387-97.

3. Sabatine MS, Giugliano RP, Keech AC, et al. Evolocumab and clinical outcomes in patients with cardiovascular disease. N Engl I Med 2017; 376: 1713-22.

4. Holmes MV, Smith GD. Dyslipidaemia: revealing the effect of CETP inhibition in cardiovascular disease. Nat Rev Cardiol 2017; 14: 635-6.

5. Swiger KJ, Martin SS. PCSK9 inhibitors and neurocognitive adverse events: exploring the FDA directive and a proposal for N-of-1 trials. Drug Saf 2015; 38: 519-26.
6. Giugliano RP, Mach F, Zavitz K, et al. Cognitive function in a randomized trial of evolocumab. N Engl I Med 2017; 377: 633-43.

7. Holmes MV. Human genetics and drug development. N Engl J Med 2019; 380: 1076-9.

8. Walker VM, Davey Smith G, Davies NM, Martin RM. Mendelian randomization: a novel approach for the prediction of adverse drug events and drug repurposing opportunities. Int J Epidemiol 2017; 46: 2078-89.

9. Swerdlow DI, Preiss D, Kuchenbaecker KB, et al. HMG-coenzyme A reductase inhibition, type 2 diabetes, and bodyweight: evidence from genetic analysis and randomised trials. Lancet 2015; 385: 351-61.

10. Holmes MV, Simon T, Exeter HJ, et al. Secretory phospholipase A(2)-IIA and cardiovascular disease: a mendelian randomization study. J Am Coll Cardiol 2013; 62: 1966-76.

11. Millwood IY, Bennett DA, Holmes MV, et al. Association of CETP gene variants with risk for vascular and nonvascular diseases among Chinese adults. JAMA Cardiol 2018; 3: 34-43.

12. Ference BA, Kastelein JJP, Ginsberg HN, et al. Association of genetic variants related to CETP inhibitors and statins with lipoprotein levels and cardiovascular risk. JAMA 2017; 318: 947-56.

13. Lyall DM, Cullen B, Allerhand $M$, et al. Cognitive test scores in UK biobank: data reduction in 480,416 participants and longitudinal stability in 20,346 participants. PLoS One 2016; 11: e0154222.

14. Ference BA, Robinson JG, Brook RD, et al. Variation in PCSK9 and HMGCR and risk of cardiovascular disease and diabetes. N Engl J Med 2016; 375: 2144-53.

15. Willer CJ, Schmidt EM, Sengupta S, et al. Discovery and refinement of loci associated with lipid levels. Nat Genet 2013; 45: 1274-83.

16. Schmidt AF, Holmes MV, Preiss D, et al. Phenome-wide association analysis of LDL-cholesterol lowering genetic variants in PCSK9. BMC Cardiovasc Disord 2019; 19: 240.

17. Rao AS, Lindholm D, Rivas MA, Knowles JW, Montgomery SB, Ingelsson E. Large-scale phenome-wide association study of PCSK9 variants demonstrates protection against ischemic stroke. Circ Genomic Precis Med 2018; 11: e002162. 